AperTO - Archivio Istituzionale Open Access dell'Università di Torino

\title{
Real-Time RT-PCR Assay for the Quantitation of Polyomavirus BK VP1 mRNA Levels in Urine
}

\section{This is the author's manuscript}

Original Citation:

Availability:

This version is available http://hdl.handle.net/2318/73947

since

Published version:

DOI:10.1007/s12033-010-9245-x.

Terms of use:

Open Access

Anyone can freely access the full text of works made available as "Open Access". Works made available under a Creative Commons license can be used according to the terms and conditions of said license. Use of all other works requires consent of the right holder (author or publisher) if not exempted from copyright protection by the applicable law. 


\section{(20) \\ UNIVERSITÀ DEGLI STUDI DI TORINO}

The final publication is available at Springer via

http://link.springer.com/article/10.1007\%2Fs12033-010-9245-x 
1Real-time RT-PCR assay for the quantitation of polyomavirus BK VP1 mRNA levels 2in urine.

3Massimiliano Bergallo ${ }^{1}$, Sara Astegiano ${ }^{1}$, Francesca Sidoti ${ }^{1}$, Samantha Mantovani ${ }^{1}$, 4Giuseppe P. Segoloni ${ }^{2}$, Rossana Cavallo ${ }^{1}$, Cristina Costa ${ }^{1}$.

$5^{1}$ Virology Unit, Department of Public Health and Microbiology; ${ }^{2}$ Renal Transplant Unit, 6Department of Internal Medicine, University Hospital San Giovanni Battista di Torino, 7Turin, Italy.

8

9M.B. and S.A. contributed equally to this work and share first authorship.

10

11Running title: Real-time RT-PCR for urine BKV VP1 mRNA.

12

13Corresponding author:

14Cristina Costa, M.D.

15Virology Unit, University Hospital San Giovanni Battista di Torino

16Via Santena 9 - 10126 Turin - ITALY

17Tel: +39(11)6705630

18Fax: +39(11)6705648

19e-mail: cristina.costa@unito.it 


\section{Abstract}

2In renal transplant recipients, polyomavirus BK can reactivate resulting in graft 3nephropathy. Screening for BK virus replication may allow for earlier intervention with 4reduced allograft loss. The measurement of urinary cell BKV VP1 mRNA for identify viral 5replication levels at risk of developing nephropathy has been proposed. In this paper the 6development, optimization, and standardization of a Taqman Real-time RT-PCR assay for 7the quantitation of BKV VP1 mRNA levels in urine is described. Subsequently, the method 8has been validated on urine specimens obtained from renal transplant recipients. The use 9of VP1 mRNA measurement as a marker for viral replication and a tool for non invasive 10diagnosis of nephropathy should be regarded with great caution, given the potentially 11 limited positive predictive value and the drawbacks associated with the complexity of the 12real-time RT-PCR assay requiring an expert well trained operator and the relatively poor 13cost-efficiency ratio.

14

15

16

17 Key words

18Polyomavirus BK; Real-time RT-PCR; VP1 mRNA; BK virus-associated nephropathy; 19renal transplantation.

\section{Abbreviations}

22BKV, BK virus

$23 \mathbf{R T}$, reverse transcription 


\section{Introduction}

2Human polyomavirus $\mathrm{BK}(\mathrm{BKV})$ is a highly prevalent virus characterized by latency in 3uroepithelial cells and other sites. In renal transplantation, in the context of intense 4immunosuppression, BKV can reactivate from latency resulting in BKV-associated 5nephropathy with interstitial nephritis and/or ureteral stenosis in $1-10 \%$ of the patients, 6leading to graft failure and return in hemodialysis in 30 up to $80 \%$ of the cases (1-3). 7Screening for polyomavirus replication, by urine and serum viral load quantitation $(4,5)$, 8may allow for earlier intervention with reduced allograft loss (2). Ding et al. (6) proposed 9the measurement of urinary cell BKV VP1 mRNA for identify viral replication levels at risk 10of developing nephropathy. Although there is no definitive cut-off for BKV VP1 mRNA 11levels diagnostic of nephropathy, Dadhania et al. (7) found that the median BKV VP1 copy 12number in renal transplant patients with BKV replication without nephropathy was 13significantly lower in comparison to that reported in renal allograft recipients with biopsy 14proven nephropathy (267 BKV VP1 copies/pg of total RNA vs. 34,000, $p=0.004$ ).

15In this paper the development, optimization, and standardization of a Taqman Real-Time 16reverse transcription (RT)-PCR assay for the quantitation of BKV VP1 mRNA levels in 17urine is described. Subsequently, the method has been validated on urine specimens 18obtained from renal transplant recipients. 


\section{Materials and methods}

\subsection{Clinical samples}

3Two-hundred-seventeen urine and 217 serum specimens from 114 renal transplant 4recipients (61 males, 53 females; men age \pm standard deviation, 56.9 \pm 9.7 years; range, 529-77) were studied. The occurrence of BKV-associated nephropathy was diagnosed by 6histopathological evaluation on kidney graft biopsy, that was collected in the presence of 7renal function abnormalities and/or on the basis of clinical features suggestive of 8nehropathy and/or rejection.

9

\subsection{Nucleic acid extraction}

11Automated extraction of total nucleic acids from urine samples was performed with the 12NucliSens easyMAG platform (bioMeriéux, Marcy l'Etoile, France). As regards total RNA, 13extracted specimens were subjected to RQ1 RNase-Free DNase digestion (Promega, 14Milan, Italy), following the manufacturer's instruction. A second automated extraction was 15performed in order to remove the DNase buffer that could interfere with the amplification. 16The absence of contaminating DNA was ascertained by PCR amplification (see section 172.3), following the second extraction, that tested negative.

192.3 Real Time PCR for BKV-DNA quantitation in serum and urine samples

20BKV DNA quantitation was performed by Real Time TaqMan PCR using a commercial kit 21(BKV Q-PCR Alert Kit; Nanogen Advanced Diagnostic, Milano, Italy) for the detection of 22the target viral gene encoding for the large T-antigen of BKV with the 7300 Real Time PCR 23System (Applied Biosystems, Foster City, CA), according to the manufacturer's 24instructions and as described previously (4). Briefly, PCR amplifications were set up in a 25reaction volume of $25 \mu \mathrm{l}$ that contained $5 \mu \mathrm{l}$ of extracted sample or negative control (sterile 
1double-distilled $\mathrm{H}_{2} \mathrm{O}$ ) or plasmid. The assay is linear in the range $10^{1}-10^{6}$ copies per 2reaction, corresponding to an absolute quantitation of $10^{3}$ copies $/ \mathrm{ml}$.

\subsection{Plasmids}

5The plasmid pBKV (ATCC 33-1 [ATCC, Manassas, VA]), containing the complete genome 6sequence of BKV (linearized using restriction enzyme BamHI) and cloned into the pBR322 7(Invitrogen, Carlsbad CA) vector, was used to determine the sensitivity and linear range of 8the PCR assay.

9The plasmid pGAPDH containing a fragment of 512 bp within the region between exon 6 10 and 8 of the housekeeping gene Glyceraldehyde-3-phosphate-dehydrogenase (GenBank 11accession no. J04038; primer F 5'-GCCAAAAGGGTCATCATCTC-3'; primer R 5'12GGGGCCATCCACAGTCTTCT-3'; probe VIC-5'-TGGTATCGTGGAAGGA-3'-MGB 13designed using the Primer Express 3.0 software (Applied Biosystems) was cloned into $14 \mathrm{pCR}^{\circledR} 2.1$ - TOPO-TA ${ }^{\circledR}$ cloning (Invitrogen) and propagated in TOP10 high-efficiency 15chemically-competent cells (Invitrogen), according to the manufacturer's instructions.

\section{5 cRNA standard production}

18A large amount of cRNA of the pBKV was obtained by using the T7 RiboMAX Express 19Large Scale RNA Production System (Promega), according to the manufacturer's 20instructions. After the transcription, the DNA template was removed by adding RQ1 21RNase-free DNase to a concentration of $1 \mathrm{U} / \mu \mathrm{g}$ DNA. After incubation at $37^{\circ} \mathrm{C}$ for $15 \mathrm{~min}$., 22the RNA was purified by performing two automated extractions with the NucliSens 23easyMAG instrument (bioMeriéux). The concentration of BKV-cRNA was estimated by 24spectrophotometric reading at $\mathrm{OD}_{260}$. To determine the sensitivity of the RT-PCR assay, 25stock preparations of BKV-cRNA were diluted to $10^{-1}$ copies/ $\mu$ l by serial 10 -fold dilutions. 
1To simulate the natural specimen, BKV-cRNA was extracted in parallel with the nucleic 2acid of the sample.

3

42.6 Reverse transcription

5The RT reaction was done by a random cDNA syntesis from $10 \mu$ of extracted sample 6RNA in two step: first a reaction mixture containing random primers (50 ng/ml)(Invitrogen) 7and $10 \mathrm{mM}$ dNTPs (Invitrogen, Carlsbad, CA) was added and incubated at $65^{\circ} \mathrm{C}$ for $5 \mathrm{~min}$. 8using the 9800 Fast Thermal Cycler (Applied Biosystems, Monza, Italy); subsequently, a 9second reaction mixture containing 0.1 M DTT, $10 \times$ Buffer (200 nM Tris- $\mathrm{HCl}$ [pH 8.4], 500

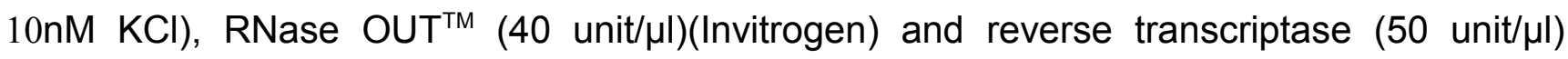
11(SuperScript ${ }^{\mathrm{TM}}$ II RT, Invitrogen), giving a total volume $20 \mu$, was used. After incubation for $1210 \mathrm{~min}$. at $25^{\circ} \mathrm{C}, 50 \mathrm{~min}$. at $42^{\circ} \mathrm{C}$ and $15 \mathrm{~min}$. at $70^{\circ} \mathrm{C}$, the cDNA mixture was used as 13target cDNA.

14

152.7 Optimization and standardization of the Real-time RT-PCR assay for BKV VP1 mRNA 16Primers and probe for BKV VP1 mRNA were obtained from the literature (6) and were as 17follows: $\quad$ primer F 5'-TGCTGATATTTGTGGCCTGTTTACTA-3'; $\quad$ primer $\quad \mathrm{R}$ 5'18CTCAGGCGGATCTTAAAATATCTTG-3'; probe 5'-FAM19AGCTCTGGAACACAACAGTGGAGAGGCC-TAMRA-3'. For the optimization of the assay, 20various conditions that affect amplification were tested, such as concentrations of primers 21(0.12 mM; $0.5 \mathrm{mM} ; 0.9 \mathrm{mM})$ and probe (0.1 mM; $0.18 \mathrm{mM} ; 0.25 \mathrm{mM})$, and $\mathrm{MgCl}_{2}(3 \mathrm{mM} ; 4$ 22mM; $5 \mathrm{mM}$ ). Two $\mu \mathrm{l}$ of cDNA, retrotranscripted BKV-cRNA standard or negative control 23(sterile $\mathrm{H}_{2} \mathrm{O}$ ) were added to $23 \mu \mathrm{l}$ of a reaction mix containing $1 \mathrm{X}$ master mix (Invitrogen), 24primers and probe, giving a final volume of $25 \mu$ l. Amplification profile was optimized for 25the 7300 Real Time PCR System (Applied Biosystems), as described above. 
1The sensitivity of Real Time PCR assays is defined as the lowest concentration of target 2quantified at a frequency of $100 \%$. The dynamic range (or linearity rate) was evaluated 3using 10-fold dilutions (from $10^{10}$ to $10^{\circ}$ copies/reaction) of BKV-cRNA standard. Precision 4or intra- and inter-assay variability (CV) was evaluated using different concentrations of 5standard BKV-cRNA (ranging from $10^{5}$ to $10^{2}$ copies/reaction) within a single run ( $\mathrm{n}=10$ ) 6or different run experiments $(n=10)$ with single replicates of each concentration 7considered as an unknown specimen. The primers were tested for potential cross8reactivity with related viral sequences of polyomaviruses JC, SV40, KI and WU based on 9the data available at the BLAST alignment software. Similarly, BLAST was also performed 10on short fragments (primers lacking at the 3'end down to 15 nucleotides).

11 In order to ascertain the identity of the resulting products a cycle sequencing was 12performed. PCR amplification products were used as templates for DNA sequencing using 13the BigDye Terminator chemistry, following the manufacturer's instructions (BigDye 14Terminator v3.1 Cycle Sequencing Kit [Applied Biosystems, Warrington, UK]). Cycle 15sequencing was performed with the 9800 Fast Thermal cycler (Applied Biosystems) under 16the following conditions: an initial denaturation step of 1 min. at $96^{\circ} \mathrm{C} ; 96^{\circ} \mathrm{C}$ for 10 sec., $1750^{\circ} \mathrm{C}$ for $5 \mathrm{sec}, 60^{\circ} \mathrm{C}$ for $4 \mathrm{~min}$ for twenty-five cycles. In order to purify the sequencing 18product from not-incorporated BigDye Terminators, the mixture was loaded direct onto 19Centrisep columns (Applied Biosystems). Subsequently, sequencing products were 20subjected to capillar gel-electrophoresis using the 3130x1 Genetic Analyzer (Applied 21Biosystems).

$22 \mathrm{~A}$ number of precautions were undertaken to prevent the occurrence of false-positive 23results. Each RT-PCR run included control reactions lacking template (no-template 24 controls) to test for the presence of contamination or the generation of nonspecific 25amplification products under the assay conditions used. The optimization criteria for the 26Real Time PCR assay for the housekeeping gene GAPDH were the same of VP1 mRNA. 


\subsection{Quantitation of the urine BKV VP1 mRNA level}

3For Real-time RT-PCR quantitation, a standard curve was created in a 4-log range by 104fold serial dilutions of the BKV-cRNA and pGAPDH. Urine VP1 mRNA levels were 5measured by normalizing the number of mRNA copies on the basis of the number of 6urinary cells. This was achieved by amplifying separately the housekeeping gene GAPDH 7 (considering the linearity range of the pGAPDH ranging from $10^{2}$ to $10^{5}$ ) and the viral 8target. In this study, a patient was classified as BKV replication positive if the urine VP1 9mRNA copy number was $>10^{3} / 10^{3}$ cells.

10

\subsection{Statistical analysis}

12The coefficient of variation for samples quantified in repeated measurements was 13calculated. Descriptive statistics were expressed as absolute value and percentage. For 14statistical analysis the $\mathrm{t}$ test was used. Operating characteristics, including sensitivity, 15specificity, negative predictive value (NPV), and positive predictive value (PPV) were 16evaluated by using a commercially available software (MedCalc; version 9.2.1.0). 


\section{Results}

\subsection{Optimization, standardization, and validation of Real Time PCR assays}

3The following primer/probe and $\mathrm{MgCl}_{2}$ concentrations were chosen: $0.25 \mathrm{mM} / 0.18 \mathrm{mM}$ and $43 \mathrm{mM} \mathrm{MgCl}$, and $0.5 \mathrm{mM} / 0.25 \mathrm{mM}$ and $3 \mathrm{mM} \mathrm{MgCl}_{2}$ for VP1 and GAPDH, respectively. 5The dynamic range of the home-made Real Time PCR for VP1 was evaluated by carrying 6out serial dilutions of BKV-cRNA (from $10^{10}$ to $10^{\circ}$ copies/reaction), and ranged from $10^{8}$ to $710^{2}$. The sensitivity of the assays was 10 copies/reaction. The precision, defined as the 8level of concordance of the individual test results within a single round (intra-assay 9precision) and from one run to another (inter-assay precision), was evaluated using 10different concentrations of BKV-CRNA, ranging from $10^{5}$ to $10^{2}$ copies/reaction, and is 11reported in Table 1.

12

\subsection{Clinical samples}

14Results are summarized in Table 2. Based on urine VP1 mRNA measurement, BKV 15replication was found in $31 / 217(14.3 \%)$ specimens from $20 / 114$ (17.6\%) patients; in 16particular, 15 specimens $(6.9 \%)$ of 9 patients $(7.9 \%)$ with levels $>10^{4}$ copies $/ 10^{3}$ cells and 5 $17(2.3 \%)$ of $3(2.6 \%)>10^{5}$. As regards viremia and viruria, BKV-DNA resulted positive (above $1810^{3}$ copies $\left./ \mathrm{ml}\right)$ in $18(8.3 \% 9$ and $23(10.6 \%)$ serum and urine specimens, respectively from $199(7.9 \%)$ and $15(13.2 \%)$ patients. A histologically confirmed diagnosis of BKVAN was 20made in two patients, both with VP1 mRNA levels $>10^{5}(66.7 \%$ of patients above this cut21off level) and with serum BKV-DNA $>1 \times 10^{6}$ copies $/ \mathrm{ml}(66.7 \%$ of patients above this cut22off level). Urine VP1 mRNA was detected in all the cases, except five, in association to 23viremia and in all the cases to viruria. Considering the highest level in each patient, 24median urine VP1 mRNA level was 31236 copies $/ 10^{3}$ cells; no difference of VP1 mRNA 25levels was found between the two patients who developed BKVAN and the highest values 
1in patients who did not developed BKVAN. Operating characteristics of VP1 mRNA 2 measurement for levels $>10^{5}$ copies $/ 10^{3}$ cells were as follows: sensitivity $100 \%$; specificity $357.1 \%$; positive predictive value $25 \%$; negative predictive value $100 \%$. 


\section{Discussion}

2In this paper the optimization and standardization of a Real-time RT-PCR assay for the 3quantitation of urine VP1 mRNA levels is described. This method was first proposed by 4Ding et al. (6) as a noninvasive and accurate tool for diagnosing BKVAN and has been 5used more recently to quantify BKV replication in monitoring renal transplant recipients, 6thus possibly representing an alternative approach to serum and urine viral load 7measurement. However, as pointed out by Nickeleit et al. (8) and Hirsch (9), some factors 8 could affect the relevance of VP1 mRNA quantitation, in particular the high susceptibility of 9the extraction of mRNA from urinary cells to erroneous results and the degree of viral 10genomic VP1 DNA contaminating the VP1 cDNA preparation, that could be overcome by 11the quantitation of VP1 signals after omitting reverse transcription or after prior DNase 12digestion. In the present study, the potential drawbacks associated to mRNA extraction 13have been overcome by performing an automated extraction of total nucleic acids, a 14DNase digestion of extracted specimens, and a second automated extraction to remove 15 the DNase buffer that could interfere with the amplification. This procedure, by removing 16viral genomic VP1 DNA contaminating potentially the VP1 cDNA preparation, should allow 17 for a more correct quantitation of BKV VP1 mRNA.

18The Real time RT- PCR assay for BKV VP1 mRNA designed by Ding et al. (6) target VP1, 19a major capsid protein responsible for the characteristics icosahedron feature and enabling $20 \mathrm{BKV}$ to entry into cells. This target was chosen because the transcription and translation of 21VP1 are contingent upon viral DNA replication, so that VP1 mRNA are detected only in 22 infected productively cells. The design of primers and probe was made to target a highly 23conserved region of the VP1 region and incorporated multiple sequence mismatches with 24the polyomavirus JCV VP1 region. The assay was optimized by examining different primer 25and probe concentrations and was found to be very sensitive with a minimum cut-off for 
1detection of 10 copies/reaction. The dynamic range was evaluated by carrying out serial 2dilutions of BKV-cRNA and ranged from $10^{8}$ to $10^{2}$. When increased sensitivity and broad 3 dynamic range are combined, it is possible to quantify template from samples containing a 4 wide range of concentrations, as in clinical samples. This avoids the need for dilution of 5the amplicon prior to conventional detection or repetition of the assay using a diluted 6sample because the first result falls outside the detection limits. The assay reproducibility 7 was high with a median intra-assay and inter-assay variability of $0.445 \%$ (range $0.31 \%$ to $80.49 \%$ ) and $1.98 \%$ (range $1.90 \%$ to $2.33 \%$ ), respectively.

9To confirm the specificity of the resulting product and that the assay did not cross-react 10 with related viral sequences of polyomaviruses $\mathrm{JC}, \mathrm{SV} 40, \mathrm{KI}$ and $\mathrm{WU}$, the primer set was 11evaluated by the BLAST alignment software and a cycle sequencing of the PCR 12amplification product was performed.

13The assay has been validated subsequently on clinical specimens from a group of renal 14transplant recipients in which two case of confirmed nephropathy was diagnosed. The 15availability of a reliable quantitative test permitted to evaluate different cut-off levels in 16relation to the presence of viral replication and the development of BKV-associated 17nephropathy. The identification of viral replication represents a tool to evaluate the risk of 18development of nephropathy, although high levels of viral replication may occur even in 19the absence of it (4). Polyomavirus BK replication occurs in the urothelial cells and results 20in shedding of urothelially derived decoy cells in high number and only replicating BKV 21leads to these cytopathic changes and the shedding of decoy cells in urine. In this study, 22the measurement of VP1 mRNA copies has been normalized on the basis of the number 23of urinary cells, rather than on the amount of total RNA. Because decoy cells mostly 24contain BK virus antigens (9), it is likely that extracted mRNA originated from these cells. 25Therefore, the availability of a RT-PCR that normalized the measurement of BKV VP1 26mRNA on the basis of the number of cells could be useful for investigating the relationship 
1between these findings and other markers of viral replication, such as urine cytology (i.e. 2decoy cells), viremia and viruria, in kidney transplant recipients at risk of BKV-associated 3nephropathy. Nevertheless, it has to be taken into account that the use of the 4housekeeping gene GAPDH as quantification control might be affected by different factors 5such as treatments and biological processes, thus potentially influencing its role in 6normalisation when considering severely altered or dead cells as shed renal tubular cells 7(10). Polyomaviruses replication may occur without clinical effects along the urothelium of 8healthy individuals and kidney allograft recipients. There are established protocols to 9screen for BKV-associated nephropathy, based on BKV-DNA detection by real time PCR 10in blood and urine $(\mathbf{4 , 1 1 )}$; these screening methods already provide a reliable basis for 11further treatment recommendation. Therefore, the use of VP1 mRNA measurement as a 12tool for non invasive diagnosis of nephropathy should be regarded with great caution, 13given the low prevalence of confirmed nephropathy in our study and the potentially limited 14positive predictive value, even considering high VP1 mRNA values, and the drawbacks 15associated with the complexity of the real-time RT-PCR assay requiring an expert well 16trained operator and the relatively poor cost-efficiency ratio. 
1Source of funding. This work was supported by a research grant by the "Compagnia di 2San Paolo" to R.C. 


\section{References}

21. Hariharan, S. (2006) BK virus nephritis after renal transplantation. Kidney Int. 69, 6553662.

42. Hirsch, H.H., Brennan, D.C., Drachenberg, C.B., et al. (2005) Polyomavirus-associated

5 nephropathy in renal transplantation: interdisciplinary analyses and recommendations.

6 Transplantation 79, 1277-1286.

73. Nickeleit, V., Singh, H.K., and Mihatsch, M.J. (2003) Polyomavirus nephropathy: 8 morphology, pathophysiology, and clinical management. Curr. Opin. Nephrol.

9 Hypertens. 12, 599-605.

104. Costa, C., Bergallo, M., Astegiano, S., et al. (2008) Monitoring of BK virus replication in

11 the first year following renal transplantation. Nephrol. Dial. Transplant. 23, 3333-3336.

125. Viscount, H.B., Eid, A.J., Espy, M.J., et al. (2007) Polyomavirus polymerase chain 13 reaction as a surrogate marker of polyomavirus-associated nephropathy.

14 Transplantation 84, 340-345.

156. Ding, R., Medeiros, M., Dadhania, D., et al. (2002) Noninvasive diagnosis of BK virus 16 nephritis by measurement of messenger RNA for BK virus VP1 in urine.

17 Transplantation $\mathbf{7 4}, 987-994$.

187. Dadhania, D., Snopkowski, C., Ding, R., et al. (2008) Epidemiology of BK virus in renal

19 allograft recipients: independent risk factors for BK virus replication. Transplantation $2086,521-528$.

218. Nickeleit, V., Steiger, J., and Mihatsch, M.J. (2003) Re: noninvasive diagnosis of BK 22 virus nephritis by measurement of messenger RNA for BK virus VP1. Transplantation $2375,2160-2161$.

249. Hirsch, H.H. (2003) VP1 messenger RNA levels in urine for diagnosing BK virus 25 nephropathy? Transplantation 75, 2160. 
110. Bustin, S.A. (2000) Absolute quantification of mRNA using real-time reverse 2 transcription polymerase chain reaction assays. J. Mol. Endocrinol. 25, 169.

311. Ramos, E., Drachenberg, C.B., Wali, R., et al. (2009) The decade of polyomavirus BK4 associated nephropathy: state of affairs. Transplantation 87, 621. 
1Figure 1. Dynamic range of BKV VP1 mRNA quantitation with the Real-time RT-PCR 2assay. Number of cycle threshold (Ct) is plotted versus copy number (from $10^{2}$ to $10^{8}$ ). 3

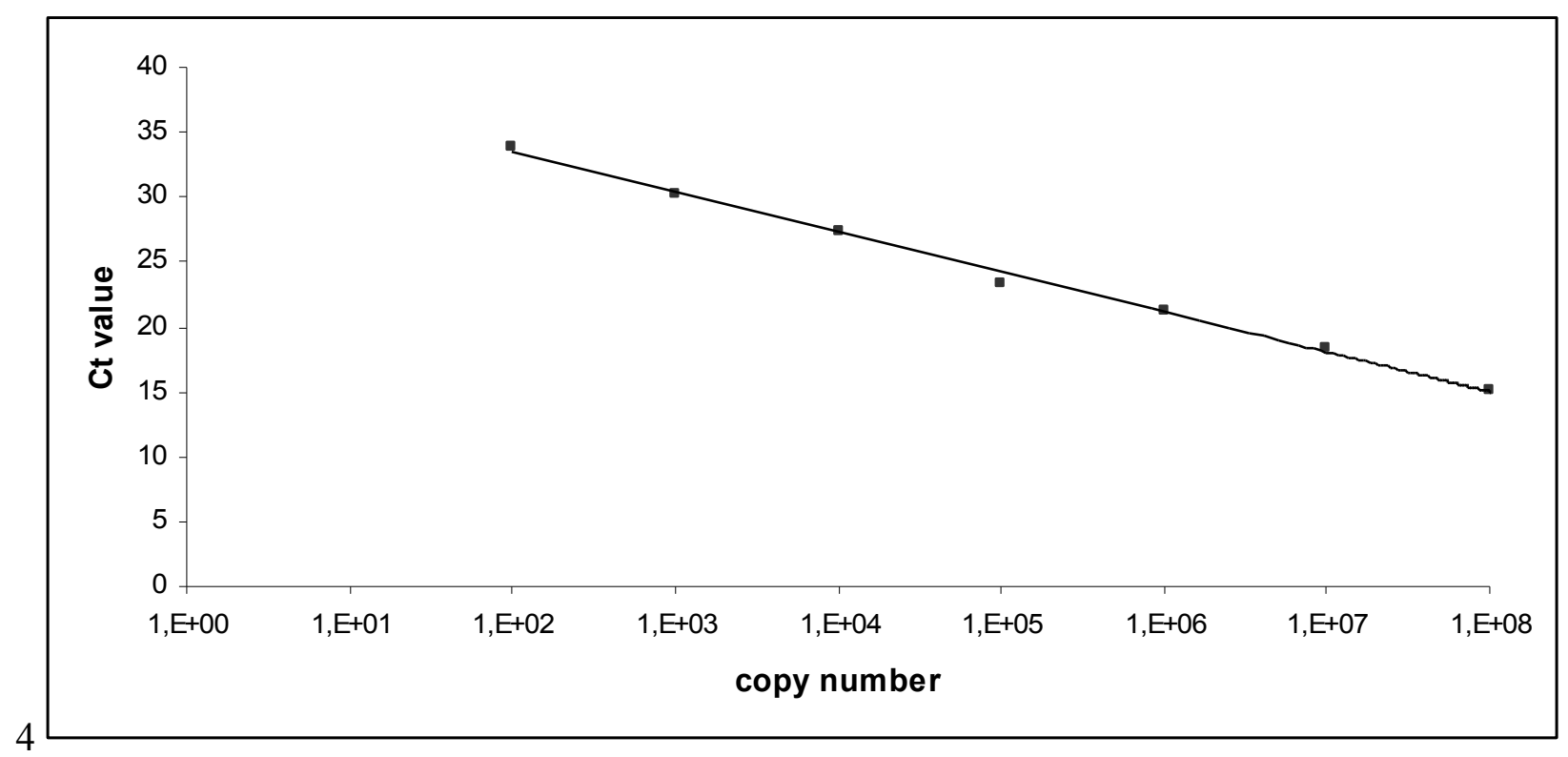


1Table 1. Intra- and inter-assay variability of standard BKV-cRNA by Real Time PCR.

\begin{tabular}{|c|c|c|}
\hline BKV-cRNA (copies/reaction) & Intra-assay variability (\%) & Inter-assay variability (\%) \\
\hline $10^{2}$ & 0.49 & 2.33 \\
\hline $10^{3}$ & 0.48 & 1.97 \\
\hline $10^{4}$ & 0.41 & 1.90 \\
\hline $10^{5}$ & 0.31 & 1.99 \\
\hline
\end{tabular}


1Table 2. Results of BKV VP1 mRNA measurements in renal transplant recipients. $2^{\star}$ Two $(66.7 \%)$ with confirmed diagnosis of BKVAN.

\begin{tabular}{|l|l|l|}
\hline \multicolumn{1}{|c|}{ Urine VP1 mRNA } & Specimens N (\%) & Patients N(\%) \\
(copies $/ 10^{3}$ cells) & Total $=217$ & Total = 114 \\
\hline$>10^{3}$ & $31(14.3 \%)$ & $20(17.6 \%)$ \\
$>10^{4}$ & $15(6.9 \%)$ & $9(7.9 \%)$ \\
$>10^{5}$ & $5(2.3 \%)$ & $* 3(2.6 \%)$ \\
\hline
\end{tabular}

4 